山्ل FRANÇAISE

$>\mathrm{DE}$

묄 PÉDAGOGIE

\section{Revue française de pédagogie}

Recherches en éducation

162 | janvier-mars 2008

Acquisitions et progressions scolaires : recherches en psychologie

\title{
Progressions scolaires, mémoire de travail et origine sociale : quels liens à l'école élémentaire?
}

Academic progress, task memory and social background. Their connections in elementary school

Progresiones escolares, memoria de trabajo y origen social : ¿qué relaciones en la escuela elemental?

Schulische Fortschritte, Arbeistgedächtnis und soziale Herkunft: wie stehen sie in Verbindung in der Grundschule

Pierre Barrouillet, Valérie Camos, Sophie Morlaix et Bruno Suchaut

\section{(2) OpenEdition}

\section{Journals}

Édition électronique

URL : http://journals.openedition.org/rfp/719

DOI : $10.4000 /$ rfp.719

ISSN : $2105-2913$

Éditeur

ENS Éditions

Édition imprimée

Date de publication : 1 janvier 2008

Pagination : 5-14

ISBN : 978-2-7342-1118-1

ISSN : 0556-7807

\section{Référence électronique}

Pierre Barrouillet, Valérie Camos, Sophie Morlaix et Bruno Suchaut, «Progressions scolaires, mémoire de travail et origine sociale : quels liens à l'école élémentaire? », Revue française de pédagogie [En ligne], 162 | janvier-mars 2008, mis en ligne le 01 janvier 2012, consulté le 10 décembre 2020. URL : http://journals.openedition.org/rfp/719; DOI : https://doi.org/10.4000/rfp.719 


\title{
Progressions scolaires, mémoire de travail et origine sociale : quels liens à l'école élémentaire?
}

\author{
Pierre Barrouillet, Valérie Camos, \\ Sophie Morlaix et Bruno Suchaut
}

Issu d'une recherche portant sur la structure et l'évolution des acquisitions à l'école élémentaire, cet article se focalise sur les liens entre les apprentissages scolaires et les capacités cognitives appréhendées par la mémoire de travail. Reposant sur une approche longitudinale, l'article vise à mettre en évidence la relation qui existe entre la mémoire de travail et les acquisitions scolaires au cours du cycle III. Le lien entre mémoire de travail et origine sociale est également examiné. Les résultats offrent des pistes de réflexion en matière de politique éducative, notamment quant au développement d'activités susceptibles de réduire les écarts sociaux et cognitifs entre les élèves.

Descripteurs (TEE) : compétence, apprentissage, origine sociale, aptitude cognitive, réussite scolaire.

\section{INTRODUCTION}

Les recherches françaises qui ont examiné les déterminants de la réussite scolaire à l'école élémentaire distinguent généralement deux catégories de facteurs. La première a trait aux caractéristiques personnelles des élèves, celles-ci étant représentées par des variables socio-démographiques et scolaires. La seconde se rapporte aux facteurs contextuels qui rendent compte de l'environnement scolaire de l'élève. Cette distinction se justifie à la fois sur le plan théorique et méthodologique. Du point de vue théorique, les interrogations varient selon que l'on considère les facteurs liés à l'élève ou au contexte d'enseignement. Par exemple, au niveau de l'élève, la variable origine sociale intéresse particulièrement les sociologues de l'éducation qui peuvent ainsi mesurer les inégalités sociales de réussite et leur évolution au cours de la scolarité (Duru-Bellat, 2002). Les facteurs contextuels peuvent être potentiellement très nombreux et les recherches effectuent de nouveau une distinction selon que ceux-ci concernent l'établissement scolaire, la classe, l'enseignant ou les pratiques pédagogiques. Sur le plan méthodologique, il importe aussi de distinguer les différents facteurs selon leur niveau d'analyse statistique : élève, classe ou établissement. 
Outre le fait de pouvoir intégrer dans une même analyse un grand nombre de facteurs, l'intérêt de ces recherches est de mobiliser une approche longitudinale qui permet de raisonner en termes de progrès réalisés par les élèves au cours d'une période donnée, généralement une année scolaire. D'un point de vue plus concret, l'estimation des progrès des élèves s'effectue par la mise en relation statistique de deux mesures des niveaux d'acquisition en début et en fin d'année. Pour rendre compte du niveau des élèves, les recherches mobilisent, soit des tests élaborés spécifiquement à cette fin, soit les épreuves des évaluations nationales (Caille \& Rosenwald, 2006). Dans tous les cas, les acquisitions des élèves sont appréhendées de façon globale, avec au mieux des découpages par discipline ou par domaine. Le niveau d'acquisition des élèves en début d'année scolaire est utilisé de façon instrumentale uniquement pour permettre de mesurer les progressions en cours d'année. II est donc systématiquement intégré dans les régressions multivariées pour raisonner "à niveau initial donné » et permettre ainsi d'isoler l'effet des différentes variables au cours d'une période précise. Or, ce niveau d'acquisition initial englobe simultanément des compétences scolaires et des habiletés cognitives sans que l'on puisse clairement les distinguer dans les épreuves administrées aux élèves. L'objectif de cet article est précisément de se centrer sur les capacités cognitives des élèves appréhendées par la mémoire de travail et d'étudier les relations que celle-ci entretient avec les facteurs les plus prédictifs de la réussite, à savoir le niveau scolaire initial et l'origine sociale. Les questions abordées portent plus précisément sur le rôle de la mémoire de travail dans les apprentissages des élèves à l'école élémentaire, cette problématique ayant été peu abordée dans le champ des sciences de l'éducation. Ces questions se déclinent globalement en deux points.

Un premier point est de s'interroger sur le lien entre l'évolution des performances scolaires et la mémoire de travail des enfants. L'approche longitudinale utilisée dans cette recherche mobilise plusieurs évaluations successives et permet ainsi d'évaluer la dynamique des apprentissages. Il est alors intéressant de savoir si le niveau de performance scolaire dépend de la même manière des capacités cognitives (mémoire de travail) à différents niveaux de la scolarité. Il est en effet possible que les apprentissages fondamentaux dépendent de ces capacités de manière plus étroite que les acquisitions ultérieures qui, elles, pourraient davantage dépendre de l'acquisition de règles ou de connaissances factuelles plus sensible aux effets des milieux éducatifs, scolaires et sociaux.
Une fois la contribution spécifique des capacités en mémoire de travail aux progressions des élèves établie, un second point portera sur la place relative des facteurs sociaux, l'origine sociale de l'élève plus exactement, comparée à celle des capacités cognitives pour expliquer les différences de progressions entre élèves. Les résultats d'une analyse portant sur la relation entre origine sociale, mémoire de travail et progrès des élèves seront alors proposés.

Après avoir défini la manière dont les capacités cognitives ont été appréhendées et mesurées dans notre travail, nous présenterons les données mobilisées avant d'exposer les résultats relevant des deux points énoncés précédemment. Pour conclure, l'intérêt de ces résultats sera dégagé, notamment sous l'angle de la politique éducative pour l'école primaire.

\section{MÉMOIRE DE TRAVAIL ET CAPACITÉS COGNITIVES}

La mémoire humaine est traditionnellement conçue comme constituée de la mémoire à long terme, dont la capacité semble quasi illimitée et qui permet de maintenir des informations sur des durées extrêmement prolongées et de la mémoire à court terme, dont la capacité est limitée et qui permet de maintenir des traces actives pendant une durée brève. Nous nous centrerons ici sur la mémoire à court terme. Elle a longtemps été considérée comme un réceptacle passif, uniquement utilisé pour stocker un nombre limité d'informations. Cependant, un certain nombre de résultats expérimentaux ont montré qu'elle servait aussi de lieu de traitement de l'information. Les tâches cognitives quotidiennes (lire un article dans le journal, calculer la somme due pour un repas au restaurant, etc.) impliquent souvent le maintien temporaire d'informations alors même que des opérations mentales sont effectuées simultanément. Ainsi, le concept de mémoire de travail (Atkinson \& Shiffrin, 1968 ; Baddeley \& Hitch, 1974) a peu à peu remplacé celui de mémoire à court terme. II est aujourd'hui utilisé pour rendre compte du système ou des mécanismes qui sous-tendent le maintien de l'information pertinente durant la réalisation de tâches cognitives.

Les premiers modèles de mémoire de travail (Daneman \& Carpenter, 1980 ; Case, 1985) reposaient sur une métaphore d'“ espace de travail ». D'après ces modèles, la mémoire de travail aurait deux limitations : spatiale (plus la quantité d'informations à retenir augmente et plus la charge cognitive sera importante) et temporelle (plus les traitements sont longs et plus 
l'information sera difficile à retenir). Cependant, les modèles les plus récents (Engle, Cantor \& Carullo, 1992 ; Just \& Carpenter, 1992 ; Cowan, 1995) relient le fonctionnement de la mémoire de travail à l'attention. Ainsi, la capacité de la mémoire de travail n'est plus considérée comme dépendante de l'espace nécessaire aux traitements, mais comme la quantité de ressources attentionnelles disponibles pour maintenir actives les informations utiles au traitement.

À partir de la métaphore spatiale des premiers modèles de mémoire de travail, des tâches ont été créées visant à mesurer la taille de l'espace que constitue la mémoire de travail. Ces tâches ont été appelées tâches d'empan de mémoire de travail. Elles ont la particularité de nécessiter le maintien en mémoire de listes de mots, de chiffres ou de lettres tout en effectuant une tâche secondaire. On parle alors de tâches d'empan complexe car deux activités cognitives sont à effectuer en parallèle. Un exemple de tâche de mémoire de travail est l'empan d'opérations («operation span » de Turner \& Engle, 1989). Dans cette tâche, le sujet doit mémoriser des listes de mots tout en effectuant des opérations arithmétiques intercalées entre chaque mot présenté (i.e., MAISON, $(4 \times 3) / 2=$ ?, CHIEN, $(5+7) \times 3=$ ?, rappel correct: MAISON, CHIEN). L'empan correspond à la plus longue liste dont le sujet peut rappeler tous les mots dans l'ordre de présentation sans avoir commis d'erreur sur les opérations. Un autre test très utilisé est l'empan de lecture ("reading span test» de Daneman \& Carpenter, 1980). II consiste à faire lire des séries de phrases présentées une à une au sujet, celui-ci devant mémoriser le dernier mot de chacune des phrases lues. L'empan est ici le nombre maximal de phrases dont le sujet peut rappeler le dernier mot après leur lecture. Dans ces tâches, il s'agit donc de maintenir des informations en mémoire afin de pouvoir les rappeler ultérieurement, tout en réalisant en même temps des activités cognitives complexes (résolution d'opérations, lecture de phrases).

Il a été montré qu'il existe une corrélation élevée entre l'empan de mémoire de travail mesuré grâce à ces tâches classiques et les activités cognitives complexes. Ainsi, l'empan de mémoire de lecture, mais aussi l'empan d'opérations, prédisent les performances en compréhension en lecture, mieux que ne le font les tâches d'empan simple en mémoire à court terme (1) (Daneman \& Carpenter, 1983). Les empans de mémoire de travail sont aussi de bons prédicteurs des performances en arithmétique (Hitch, Towse \& Hutton, 2001) ou encore en raisonnement (Barrouillet \& Lecas, 1999). De manière générale, les tâches de mémoire de travail se sont avérées d'excellents prédicteurs des performances scolaires chez l'enfant et chez l'adolescent (Gathercole \& Pickering, 2000). II a donc été suggéré que les tâches d'empan de mémoire de travail évaluent les ressources cognitives des sujets, ressources qui seraient particulièrement sollicitées dans la réalisation des tâches cognitives de haut niveau telles que la compréhension, l'apprentissage d'habiletés complexes ou le raisonnement (Gavens \& Camos, 2006).

Cependant, l'existence d'un lien entre les mesures d'empan obtenues grâce aux tâches classiques de mémoire de travail et les acquisitions scolaires apparaît quelque peu triviale. En effet, toutes les tâches classiques de mesure d'empan de mémoire de travail impliquent des activités elles-mêmes complexes (lecture, calcul) et fortement liées aux performances qu'elles prédisent (compréhension de textes, arithmétique, etc.). II n'est par exemple pas très étonnant que l'empan de lecture soit un bon prédicteur des performances en compréhension de lecture, puisque les deux doivent nécessiter des processus cognitifs communs.

Récemment, nous avons créé des tâches de mesure d'empan en mémoire de travail qui impliquent des activités beaucoup plus simples (Barrouillet \& Camos, 2001 ; Lépine, Barrouillet \& Camos, 2004). Pour construire ces tâches, les activités complexes impliquées dans les tâches classiques ont été remplacées par des activités élémentaires réputées pour leur facilité, voire leur caractère automatique, telles que parcourir la chaîne numérique ou lire des chiffres. Ainsi, dans la tâche baptisée " continuous operation span » (empan d'opérations continues), les opérations complexes utilisées dans l'empan d'opérations classique ont été remplacées par une succession d'opérations élémentaires (ajouter ou soustraire 1). Par exemple, le sujet doit effectuer à haute voix la série d'opérations « $4,+1,-1,+1,+1$ », les opérandes étant présentés successivement à l'écran, tout en maintenant en mémoire des lettres. Une autre tâche consiste simplement à faire lire au sujet des listes de chiffres (e.g., 1, 5, 3, 7, 4, 6, 2) présentés un à un pendant qu'il retient des lettres. Ainsi, contrairement aux tâches classiques de mesure d'empan, ces nouvelles tâches impliquent des activités cognitives élémentaires réputées peu coûteuses. On a cependant pu montrer que ces tâches sont en fait extrêmement difficiles, et donnent lieu à des performances de rappel plus faibles que les tâches d'empan classiques (Lépine, Barrouillet \& Camos, 2004 ; Lépine, Bernardin \& Barrouillet, 2005). 
Cette difficulté est probablement due au fait que ces nouvelles tâches entravent la mise en œuvre de stratégies sophistiquées de maintien en mémoire des listes à rappeler alors même que la tâche secondaire est effectuée. En effet, dans les tâches classiques, le sujet dispose du temps qu'il désire pour réaliser la tâche secondaire. Par exemple, dans l'empan d'opérations, le sujet peut interrompre momentanément la vérification des équations complexes qui lui sont présentées pour se remémorer mentalement les mots déjà lus, puis reprendre sans dommage le traitement et le conduire à terme. II est probable que les individus diffèrent dans leurs habiletés à mettre en œuvre de telles stratégies d'alternance entre les diverses activités à mener de front, la mémorisation et le traitement. À l'inverse, les tâches que nous avons créées entravent la mise en œuvre de ces stratégies dans la mesure où le décours temporel des traitements est déterminé par le rythme de présentation des stimuli à l'écran (deux secondes par opération dans l'empan d'opérations continues et une seconde par chiffre dans la tâche de lecture de chiffres). Ainsi, les tâches conçues permettent des mesures plus pures des capacités cognitives des sujets que les tâches classiques de mémoire de travail pour lesquelles la performance dépend à la fois des ressources cognitives du sujet et de facteurs stratégiques. Ces nouvelles tâches permettent donc de prolonger l'analyse des liens entre capacités en mémoire de travail et performances scolaires et de répondre aux questionnements présents dans cet article.

\section{DESCRIPTION DES DONNÉES}

Les données mobilisées pour cet article proviennent d'un échantillon d'élèves suivis du CE2 à la $5^{\mathrm{e}}$ dans le cadre d'une recherche plus large portant sur les acquisitions des élèves (Morlaix \& Suchaut, 2007). Nous disposons des résultats aux évaluations nationales de CE2 et de $6^{e}$, d'une mesure des acquisitions scolaires en fin de $5^{\mathrm{e}}$ et des caractéristiques sociodémographiques des élèves (2). De plus, des tests cognitifs ont été administrés en fin de $5^{\mathrm{e}}$ à un souséchantillon de quatre vingt sept élèves représentatifs de l'échantillon de départ (3). La sélection de ce sous échantillon a été réalisée en prenant comme critère principal de représentativité les scores des élèves aux évaluations de CE2, la distribution des scores de CE2 étant totalement comparable entre l'échantillon initial et ce sous-échantillon. Les tests cognitifs ont été administrés individuellement et rendent compte de deux dimensions principales des capacités cognitives. La première est la mémoire de travail mesurée par deux types d'épreuves: "reading letter span " (nommée "MT langage») et "continuous operation span » (nommée «MT nombre»). La seconde dimension est la vitesse des processus cognitifs appréhendée également avec deux types d'épreuves (vitesse de traitement et vitesse d'articulation). La vitesse de traitement a été mesurée à l'aide de deux tâches : une tâche de barrage où les enfants devaient le plus rapidement possible retrouver et barrer des cibles parmi un ensemble de petits dessins et une tâche informatisée de comparaison de nombres où les élèves devaient décider lequel de deux nombres présentés était le plus grand. La vitesse d'articulation est évaluée par le temps mis pour réciter le plus rapidement possible l'alphabet ; quatre mesures sont moyennées.

Les résultats aux évaluations nationales ont été collectés sur plusieurs années : 1999 pour le CE2 et 2002 pour la $6^{\mathrm{e}}$. En 1999 , les évaluations nationales de CE2 comportent sept champs de compétences : compréhension (41 items), outils de la langue (40 items), production d'écrits (10 items), travaux géométriques (17 items), mesure (32 items), travaux numériques (32 items) et résolution de problèmes (9 items). Les évaluations de 2002 à l'entrée en $6^{\mathrm{e}}$ comportent cent soixante quatre items (87 en français, 77 en mathématiques), répartis en huit champs : comprendre un texte, outils de la langue, production de texte, travaux géométriques, numération et écriture des nombres, traitements opératoires, problèmes numériques et traitement de l'information. En ce qui concerne notre problématique, la distinction entre les différents domaines des évaluations nationales n'est pas utile dans la mesure où notre intention est principalement d'appréhender un niveau scolaire global. Nous nous limiterons donc ici au score moyen (mathématiques et français).

Le tableau I présente les scores moyens (exprimés en pourcentage de réussite) et leur dispersion sur l'échantillon des quatre vingt sept élèves aux deux niveaux scolaires considérés.

Tableau I. - Scores des élèves aux évaluations CE2 et $6^{\mathrm{e}}(\mathrm{N}=87)$

\begin{tabular}{|l|c|c|c|c|}
\hline & Minimum & Maximum & Moyenne & Écart-type \\
\hline Score CE2 & 48 & 95 & 74 & 12,0 \\
\hline Score 6e & 36 & 96 & 74 & 12,4 \\
\hline
\end{tabular}


Tableau II. - Corrélations entre scores cognitifs et scores aux évaluations nationales $(\mathbf{N}=\mathbf{8 7})$

\begin{tabular}{|c|c|c|c|c|c|}
\hline & $\begin{array}{c}\text { MT } \\
\text { Langage }\end{array}$ & $\begin{array}{c}\text { MT } \\
\text { Nombre }\end{array}$ & $\begin{array}{c}\text { Vitesse } \\
\text { Traitement }\end{array}$ & $\begin{array}{c}\text { Vitesse } \\
\text { Articulation }\end{array}$ & $\begin{array}{c}\text { Score } \\
6^{e}\end{array}$ \\
\hline MT Nombre & $+0,44^{* \star *}$ & & & & \\
\hline Vitesse Traitement & $+0,22 * *$ & $+0,37^{\star * \star}$ & & & \\
\hline Vitesse Articulation & $-0,32^{\star \star *}$ & $-0,26$ ** & $-0,26 * \star$ & & \\
\hline Score CE2 & $+0,37^{\star \star \star}$ & $+0,50 * \star \star$ & $+0,13$ n.s. & - 0,05 n.s. & $+0,71^{\star \star \star}$ \\
\hline
\end{tabular}

n.s. : non significatif ${ }^{\star \star}$ : significatif au seuil de $5 \%^{\star \star \star}$ : significatif au seuil de $1 \%$.

Tableau III. - Scores moyens aux tests cognitifs et origine sociale ( $\mathbf{N}=87)$

\begin{tabular}{|c|c|c|c|c|}
\hline & MT langage & MT nombre & Vitesse traitement & Vitesse articulation \\
\hline
\end{tabular}

Le tableau II présente les corrélations entre les scores cognitifs et scolaires des différentes épreuves. Sans surprise, la corrélation la plus forte est relevée entre les deux scores mesurant les acquis scolaires des élèves. Parmi les différentes mesures de capacités cognitives, seuls les scores en mémoire de travail sont corrélés aux acquisitions scolaires. On ne relève en effet aucun lien entre les résultats en CE2 et en $6^{\mathrm{e}}$ et les scores de vitesse, que ce soit pour la vitesse de traitement ou celle d'articulation (4). Cette observation s'explique par le fait que les épreuves des évaluations nationales ne cherchent pas à discriminer les élèves sur des critères de vitesse de réalisation des tâches associées aux différents items. Quand on examine plus en détail les corrélations entre mémoire de travail et niveau scolaire, on observe que la mémoire de travail portant sur des épreuves de langage est davantage corrélée au score de $6^{\mathrm{e}}(+0,45)$ qu'au score de CE2 (+ 0,37). Le constat inverse est fait pour les épreuves de mémoire de travail portant sur les nombres où cette fois c'est le score de $6^{\mathrm{e}}$ qui est le moins corrélé à cette mesure $(+0,35$ et $+0,50$ pour le CE2).

II serait possible d'aller plus loin dans les relations entre les performances cognitives et les performances scolaires en examinant notamment les corrélations entre les scores cognitifs et les pourcentages de réussite obtenus dans des dimensions particulières des évaluations nationales. Cela a été effectué précisément sur les mêmes données dans une publication récente (Morlaix \& Suchaut, 2007). Les domaines les plus corrélés à la mémoire de travail concernent majoritairement le calcul mental, ce qui n'est pas surprenant dans la mesure où les activités de calcul mental font «naturellement » appel à cette capacité cognitive. À l'inverse, certaines dimensions n'entretiennent aucune liaison statistique avec les performances en mémoire de travail, il s'agit principalement de la production de texte, de la compréhension simple et du repérage spatial.

En ce qui concerne les variables socio-démographiques, nous nous centrerons uniquement sur l'origine sociale qui pourra être croisée avec les niveaux scolaires et les scores cognitifs des élèves. Les données de base font apparaître un classement des catégories sociales en neuf postes mais, compte tenu de nos faibles effectifs, un regroupement en deux catégories a été effectué. La première catégorie regroupe les vingt neuf enfants dont le père est ouvrier (qualifié ou non) ou agriculteur. Les autres élèves, au nombre de cinquante huit, relèvent des catégories moyennes et favorisées (professions intermédiaires et supérieures). À l'entrée au CE2, les élèves de milieu social défavorisé affichent un score moyen de $69 \%$ de réussite tandis que les autres élèves obtiennent un score de $77 \%$, soit une différence de huit points. À l'entrée en $6^{\mathrm{e}}$, cet écart est proche de neuf points : $68 \%$ pour les élèves de milieu défavorisé et $77,5 \%$ pour les autres. Des écarts existent également pour les scores mesurant les différentes dimensions des capacités cognitives (tableau III) mais ceux-ci sont 
plus faibles. Ainsi, pour les performances en mémoire de travail dans la dimension du langage, l'écart est de 0,52 $(+0,18-(-0,34))$, soit un demi écart-type de la distribution des score puisque l'échelle de mesure des scores cognitifs utilise la variable centrée réduite (moyenne de 0 et écart-type de 1).

Cette description des données suggère fortement que les acquisitions scolaires sont dépendantes à la fois de l'origine sociale et de certaines capacités cognitives des élèves, ces deux dimensions étant, par ailleurs, corrélées. La partie suivante examine plus en détail cette relation de dépendance.

\section{RÉSULTATS}

Les résultats seront présentés en deux temps. Dans un premier point nous rendrons compte du poids sur les progressions scolaires des élèves d'un aspect particulier des capacités cognitives, à savoir la mémoire de travail, puisque la vitesse de traitement n'est pas corrélée aux scores moyens des épreuves d'évaluation scolaire. Dans un second point, nous intégrerons aux analyses l'origine sociale des élèves et nous examinerons le lien que celle-ci entretient avec les deux autres facteurs retenus: mémoire de travail et progrès scolaires.

\section{Le poids de la mémoire de travail sur les progressions scolaires}

La méthode statistique qui semble la plus adaptée pour traiter cette question est la régression linéaire. Le principe de base de ce modèle est d'expliquer la variance d'une variable dépendante en fonction d'une ou plusieurs variables explicatives ou indépendantes. Un premier modèle de base explique la variance du score de $6^{\mathrm{e}}$ en fonction du score de CE2 (modèle M1 du tableau IV). Ce modèle nous informe que la variance du score de $6^{e}$ est expliquée pour moitié $\left(R^{2}=0,50\right)$ par les différences de scores des élèves à l'entrée en CE2. Le coefficient de 0,73 traduit la corrélation entre le score de CE2 et de $6^{\mathrm{e}}$ : un point de plus à l'évaluation de CE2 procure 0,73 points de plus à l'évaluation de $6^{\mathrm{e}}$. Le modèle M2 (tableau IV) introduit une variable supplémentaire, à savoir le score en mémoire de travail. II s'agit ici de mesurer l'influence du score en mémoire de travail (5) sur les performances scolaires à l'entrée en $6^{\mathrm{e}}$ pour des élèves ayant un score de CE2 identique. L'ajout du score de mémoire de travail à l'équation de régression apporte un gain
Tableau IV. - Modèles de régression expliquant

les progressions des élèves au cycle III, en fonction des acquisitions initiales et des capacités en mémoire de travail $(\mathbf{N}=87)$

\begin{tabular}{|l|c|c|}
\hline Variables explicatives & M1 & M2 \\
\hline Score CE2 & $+0,73^{* * *}$ & $+0,64^{* * *}$ \\
\hline Score Mémoire de travail & & $+3,10^{* * *}$ \\
\hline Constante & $19,97^{* * *}$ & $26,19^{* * *}$ \\
\hline $\mathrm{R}^{2}$ & 0,50 & 0,54 \\
\hline
\end{tabular}

n.s. : non significatif ${ }^{\star \star}$ : significatif au seuil de $5 \%^{* \star *}$ : significatif au seuil de $1 \%$.

Graphique 1. - Décomposition de la variance du score de $6^{e}$ en fonction du score de CE2 et du score en mémoire de travail

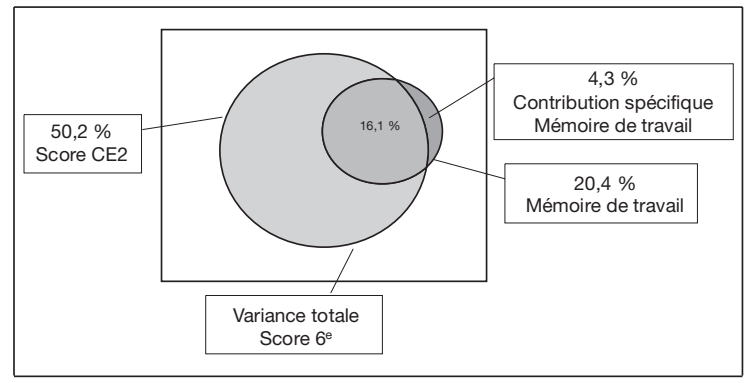

de variance non négligeable puisque le $\mathrm{R}^{2}$ passe à présent 0,54 , soit un gain de quatre points en termes de pourcentage de variance expliquée.

Le coefficient associé à la variable "mémoire de travail » est de $+3,10$ (significatif au seuil de $1 \%$ ). Cela indique qu'un point de plus au score de mémoire de travail (ce qui équivaut, compte tenu de l'échelle de mesure, à un écart-type) procure un accroissement du score de $6^{\mathrm{e}}$ de 3,10 points, à niveau scolaire de CE2 donné.

Le graphique suivant (graphique 1) permet, sur la base des modèles $\mathrm{M} 1$ et $\mathrm{M} 2$, de compléter les premiers résultats par une image de la décomposition de la variance du score de $6^{\mathrm{e}}$ en fonction du niveau d'acquisitions à l'entrée au CE2 et des performances en mémoire de travail.

Le score en mémoire de travail explique à lui seul, $20,4 \%$ de la variance du score de $6^{\mathrm{e}}$ (modèle non présenté dans le tableau IV). Comme nous venons de le noter, le modèle $M 2$, qui intègre à la fois le score 
de CE2 et le score en mémoire de travail, explique $54,4 \%$ de la variance du score de $6^{\mathrm{e}}$. Par conséquent, la contribution spécifique de la mémoire de travail à l'explication des différences de réussite des élèves à l'entrée au collège est donc de l'ordre de 4,3 points $(54,4-50,2)$ et les $16,1 \%$ restants sont donc le fait d'une part de variance conjointe entre les deux mesures de performances des élèves.

\section{Origine sociale, mémoire de travail et progrès des élèves}

Ces analyses ont été complétées en introduisant une variable supplémentaire dans les modèles précédents : I'origine sociale de l'élève (tableau V).

Le modèle M3 met en évidence l'influence de l'origine sociale sur l'évolution des acquis au cours du cycle III, puisqu'à score de début CE2 comparable, les élèves de milieu social défavorisé ont des progressions inférieures de quasiment quatre points aux autres élèves. Le modèle M4 intègre, en plus du score de CE2, à la fois l'origine sociale et le score en mémoire de travail. II fournit des résultats particulièrement intéressants. On observe en effet que l'influence de l'origine sociale est totalement absorbée par les performances en mémoire de travail. Ainsi, la variable «milieu social » n'affecte plus significativement les scores alors que le coefficient associé à la variable " mémoire de travail » reste très proche de celui relevé dans le modèle $M 2$ précédent. Ceci nous amène à nous interroger plus finement sur l'articulation entre l'origine sociale de l'élève, ses performances en mémoire de travail et ses progressions entre le CE2 et l'entrée en $6^{\mathrm{e}}$. Dans cette perspective, une analyse en pistes causales ("path analysis") a été conduite intégrant ces trois variables. Les progrès des élèves ont été appréhendés ici par les résidus de la régression entre score de 6ème et de CE2 ; une valeur positive et élevée de cet indicateur implique une forte progression des élèves entre le CE2 et la $6^{e}$, une valeur faible traduit au contraire des progressions inférieures à celles relevées en moyenne dans l'échantillon. Le graphique 2 permet de visualiser les résultats de l'analyse en pistes causales.

Le principe de cette analyse en pistes causales est de décomposer l'effet de l'origine sociale sur les progressions des élèves en un effet direct et un effet indirect. L'effet direct de l'origine sociale est nul (coefficient de régression de $-0,30$, non significatif) et confirme les résultats précédents du modèle M4. L'effet indirect de l'origine sociale sur les progrès transite par les performances en mémoire de travail.
Tableau V. - Modèles de régression expliquant les progressions des élèves au cycle III, en fonction des acquisitions initiales, des capacités en mémoire de travail, et de l'origine sociale

\begin{tabular}{|c|c|c|}
\hline Variables explicatives & M3 & M4 \\
\hline Score CE2 & $+0,69 * \star \star$ & $+0,62^{\star \star *}$ \\
\hline Score Mémoire de travail & & $+2,83^{\star \star *}$ \\
\hline $\begin{array}{l}\text { Autres professions } \\
\text { Milieu social défavorisé }\end{array}$ & $-3,99$ ** & $-3,21$ n.s. \\
\hline Constante & 24,32 *** & $29,18^{* \star *}$ \\
\hline $\mathrm{R}^{2}$ & 0,52 & 0,56 \\
\hline
\end{tabular}

Graphique 2. - Résultats de l'analyse en pistes causales entre origine sociale, mémoire de travail et progrès scolaires au cycle III

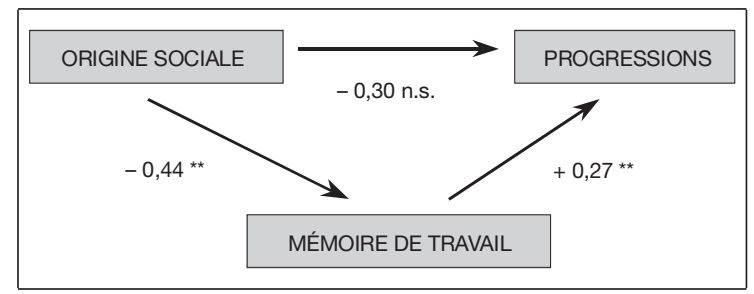

Cet effet indirect se décompose donc en deux parties. La première est symbolisée sur le graphique par la flèche qui relie l'origine sociale au score en mémoire de travail (coefficient de $-0,44$, significatif au seuil de $5 \%$ ). Ceci traduit l'effet négatif d'une origine sociale défavorisée sur les performances en mémoire de travail. La seconde partie est représentée par la flèche reliant ce même score cognitif aux progrès des élèves. Dans ce cas, le coefficient positif relevé $(+0,27$, significatif au seuil de $5 \%$ ) traduit un effet positif de la mémoire de travail sur les progrès réalisés au cycle III. L'effet indirect correspond donc au produit des deux relations précédentes, soit $-0,44 \times 0,27=$ $-0,12$.

Ces résultats fournissent des éléments sur le rôle central de la mémoire de travail dans l'explication des différences de progressions entre élèves. C'est principalement par cette dimension des capacités cognitives que l'origine sociale exerce son influence sur les progressions scolaires, sachant que par ailleurs, le rôle de la vitesse de traitement n'a pas été mis en évidence sur les données mobilisées dans cette recherche. 
Graphique 3. - Relation entre score en mémoire de travail, progrès des élèves au cycle III et origine sociale

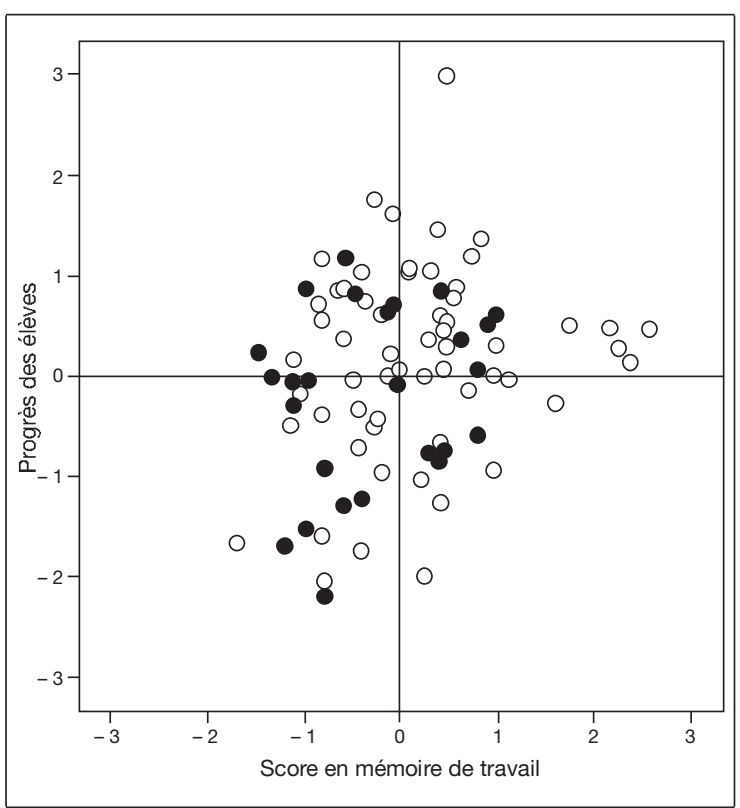

Tout cela peut donner une image assez déterministe de la réussite scolaire et il serait tentant d'avancer que les possibilités de progrès des élèves issus des milieux défavorisés seraient limitées du fait de leurs plus faibles performances en mémoire de travail. Il convient de relativiser les résultats précédents en les situant dans le contexte statistique d'ensemble des analyses. On notera en premier lieu que l'origine sociale n'explique qu'une faible part des différences de capacités cognitives, la part de variance du score de mémoire de travail expliquée par le milieu social de l'élève est proche de $7 \%$, ce qui est un chiffre modeste. On rappellera en second lieu que si les progrès des élèves au cycle III dépendent en partie de leurs capacités cognitives, cette liaison est elle-même limitée, puisque les scores en mémoire de travail expliquent également $7 \%$ des différences de progressions scolaires entre le CE2 et la $6^{\mathrm{e}}$. Le graphique suivant présente la répartition des élèves de l'échantillon en fonction des trois éléments qui viennent d'être analysés et commentés.

Les élèves sont tout d'abord distingués en fonction de leur origine sociale, les cercles noirs représentant les élèves de milieu social défavorisé. Les deux axes du graphique se rapportent d'une part aux performan- ces en mémoire de travail (axe horizontal) et, d'autre part, aux progrès réalisés entre le CE2 et la $6^{\mathrm{e}}$ (axe vertical) sur la base des scores aux évaluations. Les deux échelles étant centrées réduites (moyenne de 0 et écart-type de 1), les traits en pointillés sur le graphique matérialisent la moyenne des deux échelles.

Le graphique met en évidence la diversité des situations, et la relation faible qui lie mémoire de travail et progrès réalisés entre le CE2 et la $6^{6}$. Les élèves de l'échantillon appartenant à des milieux sociaux défavorisés se trouvent dans des positions variées. Même si, en moyenne, ils obtiennent de moins bons résultats dans les deux dimensions présentées sur le graphique (mémoire de travail et progressions), un certain nombre d'entre eux partagent leur situation avec nombre d'élèves issus de milieu social plus favorisé. Ces résultats renvoient à l'influence de l'origine sociale et des capacités cognitives, la mémoire de travail notamment, sur la réussite scolaire. En terme d'équité, il pourrait être utile d'identifier des activités qui permettraient de limiter l'influence de ces deux variables sur les écarts de progressions entre élèves. Pour cela, c'est à la fois sur le contenu de ces activités et leur modalité d'organisation concrète qu'il faut se pencher.

\section{CONCLUSION}

Les principaux résultats de cette recherche confirment le lien entre les capacités cognitives, la mémoire de travail des élèves notamment et leur aptitude à progresser dans leurs acquisitions scolaires au cours de l'école élémentaire. Les dimensions des acquis scolaires les plus liées à la mémoire de travail ont été mises en évidence à partir d'analyses en variables latentes effectuées sur les évaluations nationales de CE2 et de $6^{e}$ qui concernent un autre volet de cette recherche (Morlaix \& Suchaut, 2007). Les variables qui affichent les coefficients les plus élevés évaluent pour la plupart des habiletés en calcul mental, d'autres variables (moins nombreuses) ont trait à la production d'écrits. Cette proximité entre calcul mental et mémoire de travail n'est pas surprenante car les activités scolaires de cette nature font " naturellement » appel à cet aspect des capacités cognitives (McLean \& Hitch, 1999). À l'opposé, certaines performances scolaires entretiennent des liens très faibles avec la mémoire de travail, il s'agit de la production de texte, de la compréhension simple et du repérage spatial (mise en page d'un texte, reproduction d'une figure). 
Les résultats permettent aussi de s'interroger sur les mécanismes d'apprentissages des élèves à l'école élémentaire et peuvent déboucher sur des préconisations pertinentes en terme de politique éducative. Si le niveau d'acquisition des élèves est déterminé à hauteur de $50 \%$ par le niveau obtenu à l'entrée au CE2, les variables sociales et les capacités en mémoire de travail jouent elles aussi un rôle, certes plus limité, sur les progressions au cours du cycle III. L'originalité de notre démarche est d'avoir mobilisé des données longitudinales pour mettre en évidence le rôle de la mémoire de travail sur les résultats scolaires, tout en contrôlant l'effet de l'origine sociale. Le résultat le plus important est sans aucun doute la disparition de l'effet du milieu social de l'élève sur les progressions quand on intègre dans l'analyse les capacités en mémoire de travail. En outre, certaines dimensions des acquis scolaires, comme les habiletés en calcul mental, sont très liées à ces capacités cognitives. On peut donc penser qu'il peut être pertinent de développer certaines activités d'enseignement permettant de limiter l'influence des deux variables principales (origine sociale et capacités cognitives) sur les écarts de progressions entre élèves. Les activités mobi- lisant les habiletés en calcul mental sont donc les premières concernées et leur pratique systématique pourrait permettre de réduire le coût cognitif des activités d'apprentissage en automatisant certains processus. Dans la mesure où le niveau de compétences des élèves à l'entrée en CE2 est fortement prédicteur des progrès, c'est donc principalement avant le cycle III que doivent être mises en place ces activités systématiques afin de mieux armer les élèves dans les dimensions des acquisitions les plus prédictives de la réussite ultérieure.

Pierre Barrouillet Pierre.Barrouillet@pse.unige.ch Université de Genève

Valérie Camos valerie.camos@u-bourgogne.fr Université de Bourgogne et LEAD-CNRS

Sophie Morlaix, sophie.Morlaix@u-bourgogne.fr Université de Bourgogne et IREDU-CNRS

Bruno Suchaut bruno.suchaut@u-bourgogne.fr Université de Bourgogne et IREDU-CNRS
(1) Dans les tâches d'empan simple, les sujets doivent répéter une série d'items (chiffres, lettres ou mots) qui leur a été présentée visuellement ou auditivement. Contrairement aux tâches d'empans complexes, aucune activité secondaire n'est à mener en parallèle. Cela demande une simple mémorisation à court terme.

(2) L'échantillon concerne les élèves d'une même circonscription scolaire de l'académie de Dijon. L'ensemble des élèves des classes de CE2 (environ sept cents élèves) a fait l'objet d'un suivi longitudinal pendant cinq années et nous disposons pour quatre cents élèves des trois évaluations (CE2, $6^{\mathrm{e}}$ et $5^{\mathrm{e}}$ ).
(3) La taille de l'échantillon peut apparaître faible mais elle résulte d'une contrainte temporelle liée à la passation des tests. En effet, les tests ont nécessité une administration individuelle pouvant varier de quarante cinq à quatre vingt dix minutes par élève.

(4) Les deux scores rendant compte de la vitesse de traitement sont corrélés négativement, compte tenu de leur échelle de mesure.

(5) Seule la dimension "langage " de la mémoire de travail joue sur les progressions des élèves, le coefficient associé à la seconde dimension (nombre) n'étant pas significatif quand les deux variables sont introduites simultanément dans le même modèle.

\section{BIBLIOGRAPHIE}

ATKINSON J.R. \& SHIFFRIN R.M. (1968). "Human memory: A proposed system and its control processes ". In K.W. Spence (éd.), The psychology of learning and motivation: Advances in research and theory. New-York: Academic Press, vol. II, p. $89-195$

BADDELEY A.D. \& HITCH G.J. (1974). « Working memory ». In G.H. Bower (éd.), Recent advances in learning and motivation. New-York: Academic Press, vol. VIII, p. 647-667.

BARROUILLET P. \& CAMOS V. (2001). "Developmental increase in Working Memory Span : Resource sharing or temporal decay? ". Journal of Memory and Language, vol. XLV, $n^{\circ} 1$, p. $1-20$.
BARROUILLET P. \& LECAS J.-F. (1999). " Mental Models in conditionnal reasoning and working memory ". Thinking and Reasonning, vol. $\mathrm{V}, \mathrm{n}^{\circ}$ 4, p. 289-302.

BARROUILLET P., BERNARDIN S. \& CAMOS V. (2004). « Time constraints and resource sharing in adults'working memory span ". Journal of Experimental Psychology : General, $n^{\circ} 133$, p. 83-100.

CAILLE J.-P. \& ROSENWALD F. (2006). "Les inégalités de réussite à l'école élémentaire: Construction et évolution ». In INSEE (dir.), France, portrait social. Paris: Institut national de la statistique et des études économiques, p. 115-137.

CASE R. (1985). Intellectual development: Birth to adulthood. New-York: Academic Press. 
COWAN N. (1995). Attention and memory: An integrated framework. New-York : Oxford University Press.

DANEMAN M. \& CARPENTER P.A. (1980). "Individual differences in working memory and reading ". Journal of Verbal Learning and Verbal Behavior, $n^{\circ} 19$, p. 450-466.

DANEMAN M. \& CARPENTER PA. (1983). «Individual Differences in integrating information between and within sentences". Journal of Experimental Psychology : learning, Memory, and Cognition, $\mathrm{n}^{\circ}$ 9, p. 561-584.

ENGLE R.W., CANTOR J. \& CARULLO J.J. (1992). «Individual differences in working memory and comprehension: A test of four hypotheses ". Journal of Experimental Psychology: Learning, Memory, and Cognition, $n^{\circ} 5$, p. $972-992$

GATHERCOLE S.E. \& PICKERING S.J. (2000). «Working memory deficits in children with low achievements in the national curriculum at 7 years of age ". British Journal of Educational Psychology, $n^{\circ} 70$, p. 177-194.

GAVENS N. \& CAMOS V. (2006). «La mémoire de travail : une place centrale dans les apprentissages scolaires fondamentaux ». In E. Gentaz \& P. Dessus (éd.), Apprentissages et enseignement: sciences cognitives et éducation. Paris : Dunod, p. 91-106.

HITCH G., TOWSE J.N. \& HUTTON U. (2001). « What limits children's working memory span ? Theoretical accounts and applications for scholastic development ". Journal of Experimental Psychology: General, vol. CXXX, $\mathrm{n}^{\circ} 2$, p. 184-198.

JUST M.A. \& CAPRENTER P.A. (1992). « A capacity theory of comprehension: Individual differences in working memory ». Psychological Review, n 99, p. 122-149.

LÉPINE R., BARROUILLET P. \& CAMOS V. (2004). "What makes working memory spans so predictive of high level cognition? ". Psychonomic Bulletin and Review, $\mathrm{n}^{\circ} 12$, p. 165-170.

LÉPINE R., BERNARDIN S. \& BARROUILLET P. (2005). "Attention switching and working memory spans". European Journal of Cognitive Psychology, $\mathrm{n}^{\circ} 17$, p. 329-345.

MCLEAN J.F. \& HITCH G.J. (1999). «Working memory impairments in children with specific arithmetic learning difficulties ". Journal of Experimental Child Psychology, $\mathrm{n}^{\circ} 74$, p. $240-260$.

MORLAIX S. \& SUCHAUT B. (2007). «Évolution et structure des compétences des élèves à l'école élémentaire et au collège: une analyse empirique des évaluations nationales ". Cahiers de l'Irédu, n 68, $259 \mathrm{p}$.

TURNER M.L. \& ENGLE R.W. (1989). «Is working memory capacity task dependent? ". Journal of Memory and Language, $\mathrm{n}^{\circ} 28$, p. 127-154. 